\title{
Assessing and Changing an Organization's Innovation Culture with the Workspace Catalyst Canvas
}

\author{
Katja Thoring \\ Anhalt University of Applied Sciences, \\ Delft University of Technology \\ katja@thoring.com
}

\author{
Roland M. Mueller \\ Berlin School of Economics and Law, \\ University of Twente \\ roland.mueller@hwr-berlin.de
}

\author{
Petra Badke-Schaub \\ Delft University of Technology \\ p.g.badke-schaub@tudelft.nl
}

\begin{abstract}
The design of the workspace can be an indicator for an organization's innovation culture. This paper introduces a canvas-based collaboration tool that facilitates both, team-based analysis of existing workspaces regarding the expressed culture, and cocreating spatial design ideas with the goal to instigate a cultural change. This two-way approach is also reflected in the canvas design. We describe the development process of the canvas and its evaluation through a workshop. As a result, the contribution of this paper is twofold: (1) It informs practitioners about the relevance of spatial workspace design for cultural change, and (2) it provides insights on an atypical canvas design.
\end{abstract}

\section{Introduction}

Creatively designed workspaces have become popular among many organizations. A creative space is defined as "physical structures and elements at different scales that are deliberately designed to support creative work processes or to facilitate creativity and innovation" [20], and hence can involve pieces of furniture, the interior design, and the architectural structures of a workspace [20].

According to Schein [17], organizational culture consist of three elements: (1) visible artifacts (such as style, clothing, and the workspace design), (2) espoused beliefs and values (such as strategic goals and philosophies), and (3) underlying assumptions (perceptions, thoughts, feelings). All three aspects form the organizational culture. It can be argued that these levels can influence and reflect each other. On the one hand, the design of the physical workspace reflects the prevailing innovation culture and underlying values; on the other hand, the workspace design can also influence people's values and assumptions and hence, trigger a possible cultural change [12]. Also, Flamholtz and Randle [7] suggested that one can view organizational culture as a "corporate personality" that is defined by values, beliefs, and norms which influence the behavior of people.

However, the relationship of workspace design and organizational culture is not yet fully understood. On the one hand, the workspace can express a specific, "innovative" corporate culture, but at the same time, the space can also provide inspiration, facilitate innovation processes, and hence impact the innovation culture itself $[12,19]$. Companies might not be aware of a possible mismatch between their intended or "lived" culture and the appearance of their work environment. What is needed is a tool that would allow companies to analyze their work environment, align it with their intended corporate culture, and possibly redesign the workspace accordingly. This leads to the following research question that guided our research:

RQ: How can we design a visual collaboration tool that facilitates the assessment and the design of organizational culture as expressed by the physical workspace?

We define a visual collaboration tool as a cocreation tool that "enables and facilitates collaborative thinking, mapping, dreaming, and storytelling" [16]. It usually contains a background on which to work on [16]. One type of such collaboration tools is a canvas. According to [21], a canvas is a "two-dimensional, poster-based tool that guides a heterogenous team with a particular challenge or task. Typically, it is presented a graphical framework that decomposes a complex topic into several smaller clusters, and hence offers simplification and guidance. A canvas provides blank areas to be filled by the users, in order to invite cocreation activities and team work" [21].

This paper introduces the "Workspace Catalyst Canvas", an innovation tool to facilitate the assessment and the design of creative workspaces for their impact on corporate culture. 


\section{Methodology}

When developing the canvas, we followed the sixstep design science approach as suggested by Peffers et al. [15].

(1) We identified and formulated the problem as a hypothesis: Creatively designed workspaces are implemented in many organizations but often do not reflect the organizational culture.

(2) We defined the objectives and requirements of our intended solution: A collaboration tool (in the form of a canvas) should engage a team of employees to jointly assess their workspace and redesign it to match the existing or preferred organizational culture.

(3) We developed and designed the canvas. For the development, we identified relevant constructs that were summarized in an ontology, as suggested by [2]. For the design, we followed design principles from Gestalt Theory [1], as well as the canvas design guidelines suggested by [21].

(4) The demonstration of the developed artifact was achieved by presenting and using it at a conference workshop with 30 participants. We introduced pictures of exemplary workspaces from five real organizations from the creative sector to be assessed and redesigned by the participants.

(5) The evaluation of the canvas' usability, usefulness, and impact was conducted in a qualitative way. The workshop was analyzed by two researchers' observations, video analysis, and analysis of the resulting artifacts (the filled canvases). Moreover, we compared the results with an ex ante inquired selfassessment of the respective organizations' culture. The evaluation yielded an iteration of the canvas. Both, the ontology and the canvas design were updated according to the evaluation outcome.

(6) The communication of the research results will be achieved by scientific publications and by offering the canvas itself to interested users.

This design science approach, the resulting canvas, and its evaluation are described in more detail in the following sections.

\section{Theoretical Foundations}

\subsection{Problem formulation}

Organizational culture can be defined as the core values, assumptions, interpretations, and approaches that define an organization [4:31]. The physical work environment usually reflects an organization's style and personality [22]. Ekvall [6] identified ten factors of an organizational climate that are relevant for creativity and innovation. Among these are several factors that can be directly expressed through or encouraged by the workspace, such as playfulness, dynamism, or debate.
For example, the various offices of the global company Google show a very colorful, playful environment with games, toys, and even a slide between floors, which reflects the company's appreciation for experimentation, innovation, and risk-taking. However, according to Schein [17:27] the workspace design is only to be taken as an indicator for one possible organizational culture. Although the physical work environment is easily visible, it is difficult to decipher. The question arises, how the workspace can reflect the organizational values, and how it could be adjusted in order to match the intended culture. Miller, Casey, and Konchar [12] suggested several strategies to change an organization's culture by changing the work environment. What is missing is a collaborative tool that facilitates the process of spatial analysis, definition of the corporate culture, and of redesigning the space in order to match or change the organizational culture. The development and evaluation of such a tool is the objective of this paper.

\subsection{Objectives}

According to our problem formulation, we wanted to develop a team-based tool that would bring different stakeholders from an organization together and guide them to jointly develop a better understanding of the relationship between physical workspace and corporate culture. This tool should address both, the assessment and the design of an organization's workspace regarding the reflected innovation culture. It should allow (A) the analysis and assessment of existing workspaces, (B) agreeing on the desired corporate culture based on the identified problems and the formulation of high-level strategic goals and values, and (C) the joint development of design ideas to improve the workspace design and possibly adjust the corporate culture to match the desired outcome.

\section{The "Workspace Catalyst Canvas" for assessing and designing the workspace}

\subsection{Canvas development}

We decided to develop a collaboration tool in the form of a canvas for several reasons. According to [21], a canvas can (1) facilitate information processing (e.g. by serving as an extended memory and guide the thinking process of a team). (2) A canvas can support a team on an emotional and motivational level (e.g. by providing a platform for ideas and by triggering participation). (3) A canvas can be conducive on a social level (e.g. by creating a shared mental model, by acting as a boundary object, and by serving as a communication tool) [21]. 


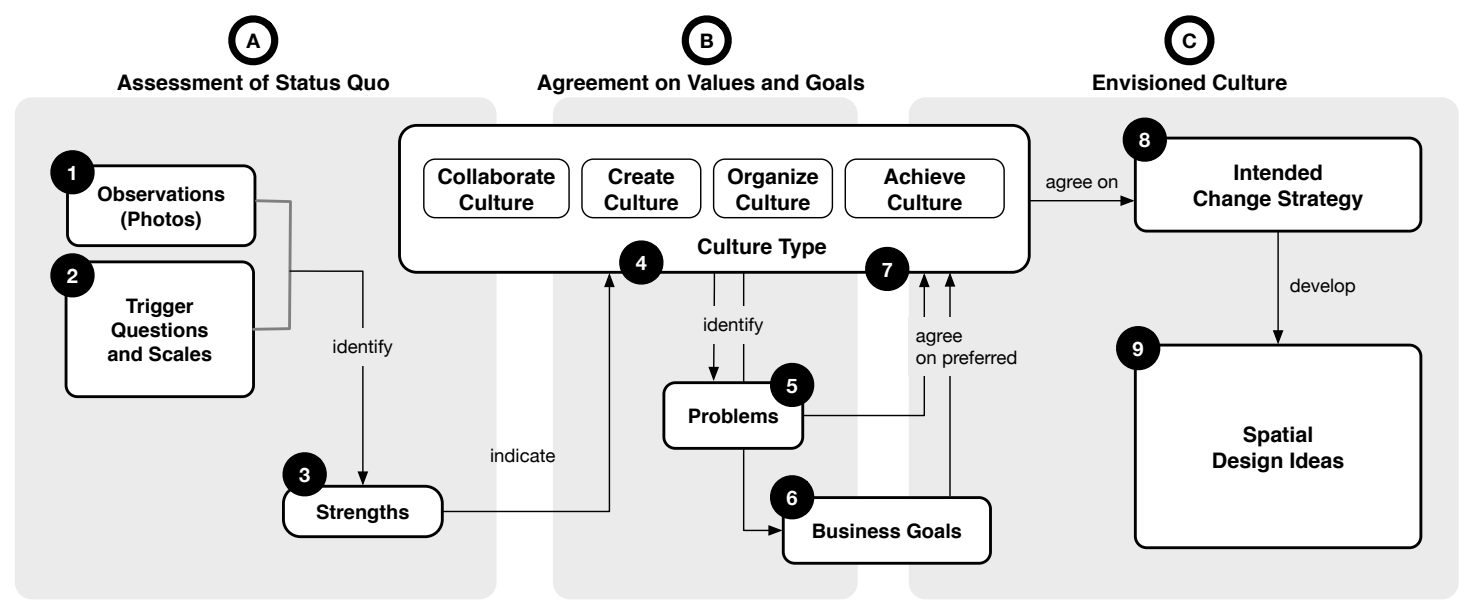

Figure 1. Ontology for canvas development (after iteration).

The development and design of our canvas is guided by Avdiji et al. [2] , who suggested three steps for developing a canvas: (1) ontology development, (2) transfer of the ontology to a visual representation, and (3) a collaboration format. Based on related theories and empirical evidence from our evaluation workshop, we identified nine relevant constructs and transferred them into an ontology (depicted in Figure 1). For clarity reasons, we show the ontology and the canvas in their final version after evaluation and iteration. The evaluation is described in detail in Section 5. The nine constructs were developed as follows.

(1) Observations. Schein [17:340] suggested a 10step process for assessing organizational culture. Step six [17:342] suggests to assess the culture through analyzing its artifacts. Hence, the first construct identified as relevant for our canvas ontology is to enable observations. We suggest providing photographs of the organizations' artifacts, as represented through the workspace.

(2) Trigger Questions. Schein [17:342] also suggests to have a consultant ask the participants questions about the artifacts and to suggest relevant categories of interest. Because the canvas is supposed to be used without a consultant, we included a set of trigger questions on the canvas itself.

(3) Strengths. Both, observations and trigger questions, would lead to identifying the strengths of the organization's culture as expressed through the workspace.

(4) Status Quo Culture Category. Based on the identified strengths, participants should be able to indicate a specific culture type as dominant in the respective organization. We refer to a framework of organizational culture types, presented by Cameron and Quinn [4] who introduced the "competing values framework" to distinguish two dimensions of organizational culture: internal versus external focus, and flexibility versus stability. The resulting quadrants between these dimensions define four types of organizational culture: (1) a collaboration culture (the "Clan") with an internal focus on human relations and flexibility, (2) a creative culture (the "Adhocracy") with an external focus on creativity, innovation, and flexibility, (3) a competitive culture (the "Market") with an external focus on competition and stability, and (4) a controlled culture (the "Hierarchy") with an internal focus on high quality, defined processes, and stability. Figure 2 depicts an adapted version of the framework, which is also included on the final canvas design.

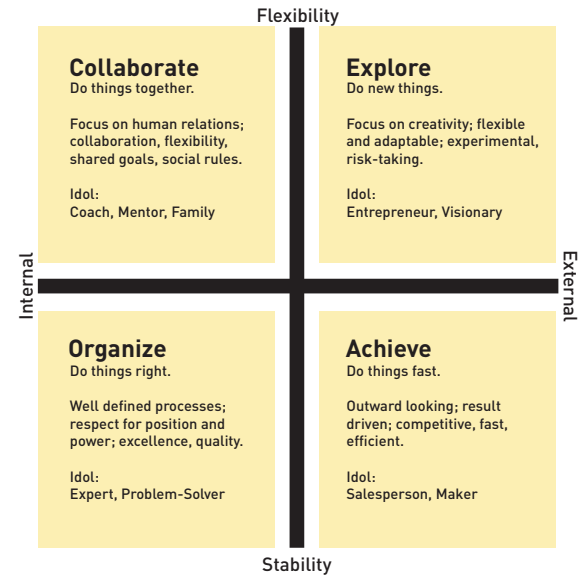

Figure 2. Iterated competing values framework [4]

(5) Problems. Any problems identified through the previous steps should be noted on the canvas to facilitate the subsequent ideation process.

(6) Business Goals. The high-level goals of an organization are usually already formulated by the company leadership [17:406]. However, we argue that it is necessary to recall these goals and note them somewhere on the canvas, in order to establish a shared mental model, before starting to develop ideas for adjusting the culture through the workspace,. 
(7) Envisioned Culture Category. One or a maximum of two of the four culture types suggested by the competing values framework [4] should be identified as the envisioned culture. Consequently, the framework should appear two times on the canvasonce for indicating the status quo, and once for agreeing on the envisioned culture category.

(8) Intended Change Strategy. These envisioned culture types might be different than the previously identified, existing ones. However, in specific cases, also an enhancement of the existing culture could be aspired; not necessarily a cultural change.

(9) Design Ideas. Building on these criteria, spatial design ideas should be developed.

These nine constructs form the basis of our ontology as illustrated in Figure 1. Constructs 3, 5, and 6 were not identified through theoretical knowledge, but empirically through our subsequent evaluation workshop, described in Section 5.

\subsection{Canvas design}

The ontology was transferred into a visually designed canvas, which was guided by the criteria for designing canvases suggested by [21]. Moreover, we followed several principles from Gestalt Theory [1] Consequently, we developed the canvas as follows:

The twofold process step of analysis and ideation was reflected by a symmetrical design of the canvas (see Figure 3). We defined three areas - the left part of the canvas is dedicated to the analysis of existing spaces, and the right part is dedicated to developing design ideas. The central part provides areas for synthesizing the identified and the envisioned culture types, as well as problems related to the existing space. This layout allows also a folding of the canvas, in cases where only one part should be addressed.

The canvas differentiates clearly between the two phases. Graphical material is usually "read" from left to right [1:33], and hence, we placed the initial assessment phase in the left and the subsequent design phase in the right area of the canvas. However, people tend to follow a so-called "Z-path" [5] when reading a graphic layout (such as a comic page or a canvas). However, this was not our intended process for filling the canvas. Instead, people should fill the left part first (from top to bottom) before moving on towards the right. This behavior is instigated by following the Gestalt principles of "proximity" and "similarity" [1]. By grouping, for example, the trigger questions and giving them a coherent design frame and color code, people would understand that these tasks were to be filled first. Similarly, the ideation area on the right was grouped as well and given a different color to indicate that these tasks were supposed to be completed later. Similarly, the central part for synthesis was aligned along a vertical axis and separated by the same color code.

To leave the users enough freedom to iteratively work with the canvas (that is, to optionally start directly with the design step), we didn't include any numbering. The canvas was designed as a poster-based printout that can be filled either by handwriting directly, of by placing Post-it notes. The sizes of the boxes for input were designed to match a standard Post-it format. Additional sticker dots could be used (alternatively to handwriting) to mark specific areas on the canvas. We included several written instructions, checkboxes, and prompts on the canvas in order to minimize additionally required facilitation. 16 Trigger questions were developed, based on existing literature about organizational culture assessment tools, such as the culture survey by Flamholtz and Randle [7], and the Organizational Culture Assessment Instrument (OCAI) by Cameron and Quinn [4]. We conceptually developed several of the presented questions further, by reframing the scope towards possible spatial relevance. For example, if the cultural characteristics for one organization were described as focused on "coordinating, organizing, and smooth-running efficiency" by the OCAI [4:26], we reframed these characteristics to the more space-related question of "to what extent does this space support correct and highquality work?". In a similar vein, we developed four questions for each of the four culture types. Presenting the trigger questions visually on the canvas should instigate a discourse and discussion among team members, which would not be possible through, for example, an online questionnaire.

We included 13 elements (building blocks or other defined input areas) on the canvas. This is significantly more than, for example, the Business Model Canvas [13], which has nine elements. This higher number was necessary, because we address two steps of the innovation process that consequently require more input sections. However, to reduce the required effort for filling the canvas, we included also various frameworks for simplified input (see next point).

We included several design specifics, mainly to simplify the complex tasks and to provide structure to the users, who otherwise might be overstrained by the number of different tasks. Specifically, we included several five-point Likert scales in the analysis area, along with trigger questions for each cultural category. This would allow people to mark their assessment of a given space by placing stickers or manually checking the respective scale. Additional checkboxes were included in the design area (right), where people could indicate the envisioned change their developed ideas would cause, according to the four culture types. We distinguish between "enhancing", "reducing", or 


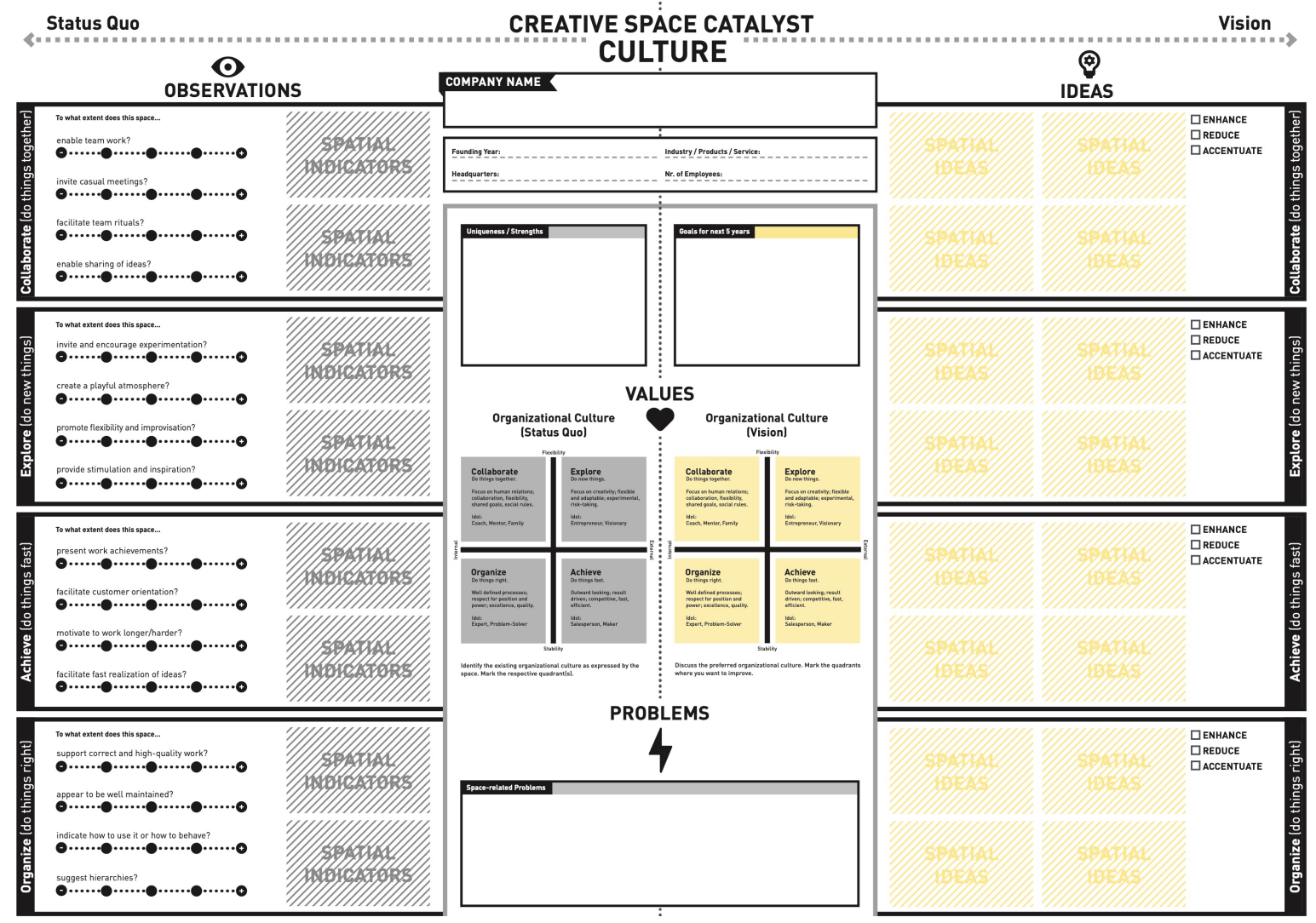

Figure 3. Culture Catalyst Canvas (second version after iteration).

"accentuating" one of the four suggested culture types, where "accentuating" resembles a more neutral strategy. Focus should be given to the respective areas that address the culture types identified as problematic (and hence should be reduced) or preferred (and hence should be enhanced). Moreover, we integrated icons, to indicate the main areas of concern, and a color-code was chosen to distinguish the two main phasesanalysis (left, grey) and design (right, yellow). Additional fields for metadata, such as company name, number of employees, etc., were included in the top area of the canvas (but not considered as separate "building blocks"). Figure 3 depicts the final canvas (after iteration), while Figure 4 gives an impression of the first version of the canvas that was used in the evaluation workshop.

\subsection{Originality of the proposed canvas}

The developed "Workspace Catalyst Canvas" is novel and peculiar in several ways. The 2-sided layout allows a flexible usage. The canvas can be folded in the middle when only the analysis or only the design step are to be followed. The applied color-coding makes the two process steps easily accessible for the participants. The integrated frameworks, checkboxes, and scales allow for a systematic and directed data input and easier analysis. For example, the Likert scales lead to a visual categorization of culture types, easily visible for the participants, which might reduce lengthy discussions. The Post it-sized boxes allow for custom-fit placement of ideas, which also results in a structured and ordered completion of the canvas. Trigger questions are printed directly onto the canvas in order to instigate team discussions.

The potential of those design features, such as color-coding and integrated frameworks, are rarely used in current canvas design [21], although such features might simplify and facilitate the allocation of particular information.

When comparing our proposed canvas with two existing canvases that address organizational culture, several differences can be observed. The "Culture Canvas" [23] consist of ten standard building blocks with additional icons. These ten blocks all address culture from a people-centered perspective. The corporate environment is not addressed at all. The "Culture Map" [9] uses a very simple swim lane layout consisting of three areas called "outcomes"; "behaviors", and "enablers and blockers". Trigger questions prompt the users to enter specific input. The canvas does not involve any visuals. Within the 
"enablers and blockers" section, also one question addresses the workspace.

\section{Canvas evaluation}

\subsection{Procedure}

In order to evaluate the usability and impact of the canvas, we conducted a workshop at a scientific design research conference in early 2018. We hosted approximately 30 participants, mainly from a design research and academic context, that were assigned randomly to six teams of 5-6 people, each. The workshop lasted 90 minutes and was facilitated by five coaches.

The workshop started with an introduction that outlined the theoretical background and our previous research on creative spaces. The main workshop activity was then conducted in small groups of five people, each. We provided a printout of the canvas, as well as Post-it notes (matching the size of the canvas boxes), pens, and a set of sticker dots ( 2 sizes and colors). Each team received a different set of 10-12 photographs, depicting the workspace of a real organization from the creative sector. Table 1 outlines the five organizations for which we provided workspace pictures. Five coaches were present to explain how to use the canvas, in case that any questions occurred.

This workshop setup is comparable to a realistic scenario in which people from a real organization would assess and redesign their own workspace. The only difference were the participants, who were not familiar with the provided spaces and the organization's context, and who also might not be as involved and engaged as they would be with their own company's workspace. But also in a realistic context, we suggest to prepare printed pictures of the workspaces to be analyzed and designed. This procedure would allow the group to work focused with the canvas, instead of having to move through the building in order to investigate different areas in person. Although the provided photos would not provide insights on non-visible aspects of the workspace (such as smells and sounds), we argue that this medium would still suffice for the intended workshop purpose. The focus of the workshop is not to assess the quality of the space, but only the visible corporate culture of the workspace, which can be sufficiently inferred from the pictures. In a realistic context, a focus group composed of different stakeholders from the respective organization would complete the canvas together.

Table 1 shows the five organizations that were selected to provide some sort of variety among the presented picture sets: the range of different workspaces included design practice (IDEO) design education (SAIC), and a startup incubator (TUM), as well as three innovation departments of three globally operating corporations from different fields, including furniture (Steelcase), automotive (MHP) and software development (SAP). These organizations either had a design-focus or were innovation departments of companies from other-non design-related - fields. Hence, we considered them from the "creative sector".

Table 1. Overview of included organizations.

\begin{tabular}{lll} 
\# & Organization & Description \\
\hline 1 & IDEO & $\begin{array}{l}\text { Globally operating design agency, US } \\
\text { origin, Munich office }\end{array}$ \\
\hline 2 & SAP & $\begin{array}{l}\text { Global Software Company, "App House" } \\
\text { Innovation Department, Berlin office }\end{array}$ \\
\hline 3 & MHP & $\begin{array}{l}\text { Porsche Digital Innovation Lab, Berlin } \\
\text { office }\end{array}$ \\
\hline 4 & Steelcase & $\begin{array}{l}\text { Global furniture manufacturer; US origin, } \\
\text { Munich office }\end{array}$ \\
\hline 5 & SAIC & Art and Design School, Chicago, US \\
\hline 6 & TUM & $\begin{array}{l}\text { University-affiliated incubator and Startup } \\
\text { Lab of TU Munich }\end{array}$ \\
\hline
\end{tabular}

The pictures were taken with permission of the respective organizations by one of this paper's authors during on-site visits of the workspace. Through a short 4-question survey among management-level employees of each organization, we were able to categorize each institution's assumed corporate culture, for later comparison with the workshop participants' assessments. For confidentiality reasons, these assessments cannot be published here, though.

The evaluation was conducted according to the following procedure: (1) The coaches took notes of their observations, during and right after the workshop. (2) The entire session was video-recorded with a wideangle lens, which allowed us to review the whole workshop afterwards, but not to look into details within the individual teams. (3) the presentation of each team, as well as the subsequent discussion was videorecorded. (4) The developed artifacts (the completed canvases) were photographed and collected for later analysis.

We analyzed the data as follows: The coaches' field notes were recorded in a database (Excel spreadsheet); videos of the groups' presentations were reviewed by two of this paper's authors. Selected observations and emerging insights were also recorded in the database. And finally, the five completed canvases were reviewed by two of this paper's authors.

Through triangulation of these different data sources we were able to evaluate the canvas on two levels: (1) the usability and comprehensibility of the canvas, and (2) the possible impact of the canvas, based on the developed design ideas and a comparison of the organization's self-assessed culture with the 
assessment of the participants. Both aspects are discussed in the following sections.

\subsection{Evaluation results regarding the canvas design and usability}

The first part of our workshop evaluation deals with the question of how participants worked with the canvas and whether they were able to understand the different canvas areas, the provided tools, and the goal. Figures 4, 5, and 6 show an exemplary filled canvas and some details. Based on the triangulation of the different data sources, the following insights could be derived:

1. High engagement was visible for all teams. Vivid discussions emerged about the shown pictures.

2. Rating of spaces on Likert scales through stickers caused team members to participate and to look thoroughly into the pictures.

3. Phrasing of the trigger questions was sometimes too negative for some culture types (e.g. visibility of "strong hierarchies"), which resulted in some types being avoided; consequently, we rephrased the questions for the second iteration of the canvas to make them all appear less negative.

4. Participants had problems to understand some terms printed on the canvas, such as "Adhocracy". Therefore, we decided to replace those labels with more common names, while still keeping the original meaning.

5. The handling of stickers, specifically the marking on Likert scales according to the trigger questions, was performed quickly and easily.

6. The two-sided canvas and color-code was understood by everybody immediately. The possibility to switch back and forth between the defined requirements on the left and the developed solutions on the right appeared to be beneficial to the process.

7. The integrated frameworks accelerated the process; all groups completed the assessment of the space faster than the allocated 20 minutes.

8. An area for framing the identified problems was missing. This could be inferred from people writing and summarizing the identified problems directly on the canvas.

9. We observed numerous discussions about the general high-level goals and values of the organization. A space to make these visible on the canvas appeared to be missing.

10. The "Actions" area for summarizing one selected solution (central lower part of the canvas) appeared not to be useful. People were simply shifting some Post-its from the right area (see Figure 4), instead of elaborating one idea.
11. Post-it-sized boxes were filled almost completely on all canvases. The provided areas seemed to motivate people to fill in the slots in order to make the canvas look "complete" [21].

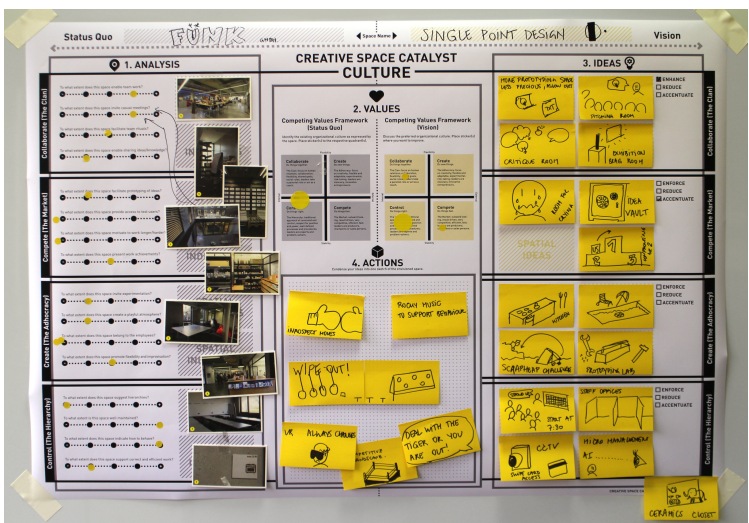

Figure 4. Exemplary filled canvas, before iteration

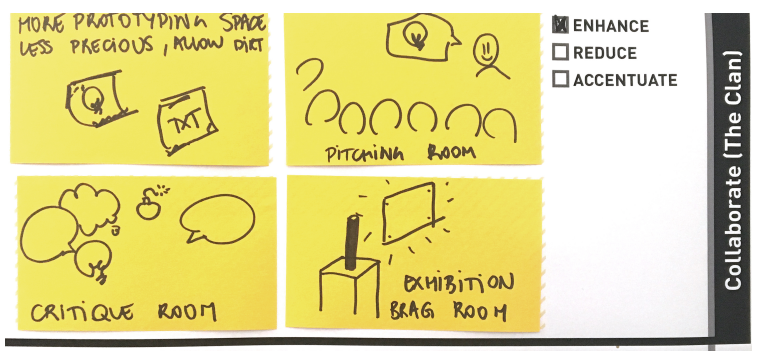

Figure 5. Filled canvas (detail).

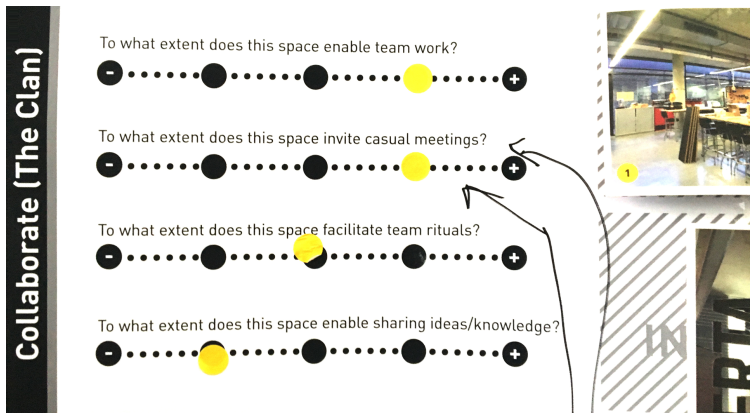

Figure 6. Filled canvas (detail).

\subsection{Evaluation results regarding the canvas impact}

The second part of our workshop evaluation deals with the actual outcomes of the workshops, which would provide us with insights on the possible impact and usefulness of the canvas. However, we acknowledge that further research is required to be able to make statements about a long-term impact of the canvas.

The assessment phase yielded several insights on how the participants evaluated the shown pictures. In two cases, the assessment of the shown spaces by the workshop participants was quite different than the 
estimated culture by the organizations themselves. For example, two of the included organization, which had rated themselves as Collaborative (dominant) and Explorative (secondary) was assessed by the participants as Organized (dominant) and Competitive (secondary) - thus quite the opposite. Although these results constitute an individual opinion and hence provide only a snapshot of the reality, they indicate a possible mismatch between an organizations' selfassessment and an external observer's impression of the workspace. Moreover, it demonstrates the relevance of the workspace design. Organizations might not be aware of how their spaces are perceived by others and that they possibly not appropriately reflect the actual corporate culture. Consequently, we argue that the canvas provides a valuable tool for companies to evaluate whether their workspace designs corroborate with their intended corporate culture.

With regards to the design phase, the workshop also yielded several insights. For the most part, the design ideas developed by the teams were supported by visual sketches (Figure 6). Among the developed solutions were several that could be considered more "fun" and "wild" ideas. One example of this category is "the room for crying" to accentuate the achievement culture. Other ideas were more down-to earth, like a room specifically designed for "standup meetings", to reduce hierarchies, and the "exhibition brag room", where everybody would find a place to show off their work. These results warrant the assumption that the canvas helps to come up with new ideas for spatial designs in order to express or change an organizations' corporate culture. We also infer that working with the canvas will help organizations and employees to raise their awareness and sensitivity to spatial design decisions.

\subsection{Canvas Iteration}

After the workshop, both, the ontology and the canvas design were slightly updated and modified, based on the results of the workshop evaluation:

1. The "Actions" area was removed.

2. The central part of the canvas was updated to let people summarize the analysis process and agree on strategic goals. A new "Uniqueness/Strengths" box was added to summarize positive aspects regarding the workspace and culture; a new "Goals" box was added to agree on high-level business goals; and a new "Problem" box was added, to let people specifically agree on and formulate what problems they see in the currently expressed culture and workspace. This area would allow them to specify what should be changed, and hence, give more focus to the ideation phase.
3. The trigger questions were rephrased to provide a wider range of issues within each category.

4. The labels of the competing values framework [4] were slightly changed to make them more comprehensible and to reduce the negative undertone of some original labels: The "Adhocracy" culture was changed to "Explore" culture; the "Market" culture was changed to "Achievement" culture; the "Hierarchy" culture was changed to "Organized" culture; and the "Clan" culture was changed to "Collaboration" culture.

Figure 3 depicts the updated, final canvas design with the updated labels and the new trigger questions. The initial version of the canvas, before iteration, is shown in Figure 4.

\section{Discussion}

\subsection{Implications}

According to Thoring et al. [21], who evaluated a total of 68 existing innovation-related canvases, most canvas designs (57 out of 68) follow the typical building-block structure that is known from the Business Model Canvas [13]. They found only 11 canvases that incorporated different shapes and layouts, other than rectangular boxes. Only a few canvases suggest a two-way usage, like we did. The Value Proposition Canvas [14] depicts a face (representing the customer) and a giftbox (representing the value proposition). Both elements are supposed to be completed iteratively in order to make the value proposition match to the customer. The Data Innovation Board [10] provides three areas that resemble the typical design thinking process of "explore", "ideate", and "evaluate" [3]. While the standardized building block design of a canvas has certainly some merits (e.g. because familiar visual structures might improve recognition time [18]), we argue that a more visual design has also its advantages.

The canvas presented in this paper is different from the typical building blocks, because its two-sided layout addresses two different perspectives on one topic. More specifically, it facilitates (1) the assessment of a given space, as well as (2) the design of a new space regarding the corporate innovation culture. It allows a flexible approach from two different starting points. Hence, the canvas can serve as (1) an assessment tool, and (2) a design tool, at the same time. We argue that the two-way design of the suggested canvas provides an increased flexibility. It is possible to work on only one half of the canvas (and optionally fold it for this purpose) in order to either assess the existing space without redesigning it, or, by contrast, to develop design ideas from scratch, without assessing an existing space. The latter scenario could 
apply, for example, to a start-up that wants to move into a new office space.

We decided not to develop two separate canvases for both purposes - one for assessing the workspace and one for designing it - because we argue that both procedures often go hand in hand. Having both steps on one canvas allows to make direct connections from an existing spatial characteristic to a preferred one. Identified problems are visible on the canvas, which makes it easier to address them in the subsequent design step. In our workshop we could not observe any difficulties of the participants when working with different tasks on one canvas. On the contrary, being able to go back to the analysis phase while working on ideas appeared to be beneficial.

Other peculiarities of the canvas design include the various integrated evaluation metrics (2-by-2 frameworks, Likert scales, and checkboxes) and the integrated trigger questions. These specifics have been found conducive to the workshop course. Participants could easily mark their assessments, as well as goals and identified problems. These formalized visualizations can accelerate the transfer of knowledge, because they add structure and eliminate misunderstandings (e.g. caused by illegible handwriting).

For organizations, the introduced "Workplace Catalyst Canvas" provides a collaboration tool that allows teams to jointly explore possible problem areas within the work environment. Adding to that, the canvas helps organizations when generating new ideas for workspace designs to instigate a cultural change.

\subsection{Limitations and future work}

The main limitation of this study is that we conducted the evaluation workshop with unrelated conference scholars, rather than in a realistic corporate setting. The use of exemplary pictures results in a lack of context about the assessed workspaces. One could argue that, as a consequence, people might have been not as thorough and engaged as they possibly would with analyzing their own space. We would expect different results and more in-depth reflection of the participants, when people were dealing as a team with analyzing their own organization.

However, when evaluating their own work environment, employees might also be less objective than the unrelated workshop participants. Future work will focus on conducting a realistic workshop with actual employees of a company to corroborate these assumptions.

Moreover, the slightly negative undertone of certain trigger questions and the labels of the competing values framework (as suggested by the original authors, Cameron and Quinn [4]) might have led to a certain resistance of the participants against these categories and hence, the results might not authentically reflect the actual estimations of the identified culture. Consequently, another workshop with the updated canvas (in which we established a more positive undertone for all four culture categories) will be conducted in the future. This workshop will test whether the same workspaces would be assessed differently, using the updated canvas.

Finally, we would like to emphasize that the workspace design is to be taken as an indicator for only one possible organizational culture. As stressed by Schein [17:27], although the physical work environment is easily visible, it is difficult to decipher. It can be dangerous to interpret the underlying cultural values and assumptions only based on the visible artifacts, because these might only reflect one's own perceptions and preconceptions.

The assessment of the workspace pictures by external workshop participants, who had no deeper insights in the context of the organization, has to be taken with caution. Hence, the results of a space's assessment are not to be taken as a definite conclusion, but rather as a hint for possible problems and potentials for improvement. Nevertheless, they can provide organizations with valuable insights and feedback on the perception of their workspaces and possible mismatches between intended and perceived cultural self-expression of the work environment. As suggested by Garland [8] a creatively designed work environment can serve as a recruitment factor to attract new employees. Hence, a better understanding of the external perception of the work environment and its related innovation culture is critical.

Finally, we acknowledge that the physical workspace is only one aspect of the organizational culture. Other aspects, such as personal freedom, idea support, trust, and available time [6] might be equally, or even more relevant to establish a corporate innovation culture. Simply redesigning the workspace will certainly not suffice, if other aspects of the organizational culture are not updated accordingly. Mckendry [11] argues that a fun work environment cannot compensate for a possible lack of company culture and "real" incentives, such as yearly cash bonuses, personal growth opportunities, or healthcare plans.

\section{Conclusions}

In this paper, we introduce a novel innovation canvas that can be used to assess and design an organization's workspace regarding the expressed corporate innovation culture. We tested the canvas in a workshop with 30 participants from design research 
and academia, who evaluated given pictures from real organizations. Their assessments regarding the innovation culture as expressed through the visible workspace designs, were compared to the self-assessed corporate culture of the respective organizations. Moreover, we analyzed the workshop course and the developed design ideas through triangulation of observations, artifact analysis, and video-recordings.

Consequently, the contribution of this paper is twofold: (1) The insights from the workshop evaluation indicate that organizations might not be aware of the external image their workspace designs can reflect, and that this image might differ from the actually "lived" corporate culture. The introduced canvas can help organizations to better assess the innovation culture that is expressed through their workspace, and to redesign their space accordingly. Moreover, the work presented in this paper stresses the relevance of the workspace design for the purpose of attracting and motivating current and future employees. (2) The results from the canvas design inform the research community about possible design options when designing a canvas. Deviating from the standard "building-block" design by including visual elements, color-codes, and assessment frameworks can provide potentials for accelerating the workshop course and adding focus.

The introduced canvas is considered a first step towards understanding the role of workspace design for an organization's innovation culture. Future research will include additional evaluation workshops to develop the canvas further and to test it in a real-life scenario.

\section{References}

[1] Arnheim, R., Art and visual perception: a psychology of the creative eye, UCA Press, Berkeley, 1974.

[2] Avdiji, H., D. Elikan, S. Missonier, and Y. Pigneur, "Designing Tools for Collectively Solving Ill-Structured Problems", Proceedings of the 51st Hawaii International Conference on System Sciences (HICSS), (2018), 400-409.

[3] Brown, T., "Design Thinking", Harvard Business Review 86(6), 2008, pp. 84-92.

[4] Cameron, K.S., and R.E. Quinn, Diagnosing and Changing Organizational Culture: Based on the Competing Values Framework, Wiley \& Sons, San Francisco, 2006.

[5] Cohn, N., "Navigating Comics: An Empirical and Theoretical Approach to Strategies of Reading Comic Page Layouts", Frontiers in Psychology 4, 2013.

[6] Ekvall, G., "Organizational climate for creativity and innovation”, European Journal of Work and Organizational Psychology 5(1), 1996, pp. 105-123.

[7] Flamholtz, E., and Y. Randle, Corporate culture the ultimate strategic asset, Stanford Business Books, CA, 2011.
[8] Garland, T., "Making a Statement with Office Space: Finding Workplaces that Last", Area Development, 2013. http://www.areadevelopment.com/workplace-trends/Q42013/workplace-trends-help-retain-attract-talent261718156.shtml

[9] Gray, D., "The Culture Map", 2015. http://www.xplaner.com/culturemap/

[10] Kronsbein, T., and R. Mueller, "Data Thinking: A Canvas for Data-Driven Ideation Workshops", Proceedings of the 52nd Hawaii International Conference on System Sciences, (2019), 561-570.

[11] Mckendry, A., "Top Incentives to Attract Top Talent", FIrefish Blog, 2018. https://blog.firefishsoftware.com/topincentives-attract-retain-talent

[12] Miller, R., M. Casey, and M. Konchar, Change Your Space, Change Your Culture: How Engaging Workspaces Lead to Transformation and Growth, John Wiley \& Sons, New York, 2014.

[13] Osterwalder, A., and Y. Pigneur, Business Model Generation: A Handbook for Visionaries, Game Changers, and Challengers, Wiley, Hoboken, NJ, 2010.

[14] Osterwalder, A., Y. Pigneur, G. Bernarda, and A. Smith, Value proposition design: how to create products and services customers want, Wiley, Hoboken, NJ, 2014.

[15] Peffers, K., T. Tuunanen, M.A. Rothenberger, and S. Chatterjee, "A design science research methodology for information systems research", Journal of management information systems 24(3), 2007, pp. 45-77.

[16] Sanders, E.B.-N., "Generative Tools for Co-designing", In S.A.R. Scrivener, L.J. Ball and A. Woodcock, eds., Collaborative Design. Springer London, 2000, 3-12.

[17] Schein, E.H., Organizational culture and leadership, Jossey-Bass, San Francisco, 1992.

[18] Sprague, D., and M. Tory, "Exploring how and why people use visualizations in casual contexts: Modeling user goals and regulated motivations", Information Visualization 11, 2012, pp. 106-123.

[19] Thoring, K., P. Desmet, and P. Badke-Schaub, "Creative environments for design education and practice: A typology of creative spaces", Design Studies 56, 2018, pp. 54-83.

[20] Thoring, K., P. Desmet, and P. Badke-Schaub, "Creative Space: A Systematic Review of the Literature", Proceedings of the 22nd International Conference on Engineering Design (ICED19), Design Society (2019).

[21] Thoring, K., R.M. Mueller, and P. Badke-Schaub, "Exploring the Design Space of Innovation Canvases", Proceedings of the Academy of Design Innovation Management Conference (ADIM), (2019).

[22] Turner, G., and J. Myerson, New workspace, new culture: office design as a catalyst for change, Gower Publishing, Ltd., 1998.

[23] Weijers, K., "The Culture Canvas", 2015. https://culturecanvas.biz 